\title{
Lesão de ombro após a vacinação: Uma revisão sistemática
}

\section{Shoulder Injury after Vaccination: A Systematic Review}

\author{
Paul J. Cagle Jr. ${ }^{10}$ \\ ${ }^{1}$ Departamento de Cirurgia Ortopédica, Icahn School of Medicine at \\ Mount Sinai, Nova York, Nova York, Estados Unidos \\ Rev Bras Ortop 2021;56(3):299-306.
}

\begin{abstract}
Endereço para correspondência Paul J. Cagle Jr, MD, Assistant Professor, Associate Program Director Department of Orthopaedic Surgery, Icahn School of Medicine at Mount Sinai, New York, NY, United State (e-mail: PauljCagleMD@gmail.com).
\end{abstract}

\section{Resumo \\ Palavras-chave \\ - bursite \\ - impacto no ombro \\ - dor em ombro \\ - vacinas contra influenza}

\section{Abstract}

recebido

20 de Maio de 2020

aceito

16 de Setembro de 2020

Publicado on-line

Dezembro 16, 2020
As reações adversas às injeções de vacina tendem a ser brandas e são incrivelmente raras. No entanto, vários casos de eventos em ombros, como bursite, dor generalizada ou diminuição da amplitude de movimento, foram relatados após vacinações de rotina. Esses eventos são conhecidos como lesões em ombro relacionadas à administração de vacina (SIRVA, do inglês shoulder injury related to vaccine administration).

Uma revisão sistemática da literatura foi realizada para identificar todos os relatos publicados de SIRVA. Vinte e sete artigos que relataram um ou mais casos de SIRVA foram encontrados. A vacina mais comumente citada foi a vacina contra influenza. Os sintomas mais comuns foram dor com início em até 48 horas e perda da amplitude de movimento do ombro. As modalidades de tratamento mais comuns foram fisioterapia, injeções de corticosteroides e administração de medicamentos anti-inflamatórios; alguns pacientes, porém, precisaram de cirurgia. Independentemente da intervenção, a grande maioria dos casos apresentou melhora da dor e da função, à exceção dos pacientes com lesão nervosa.

A SIRVA tem múltiplas possíveis etiologias, inclusive comprimento da agulha, lesão mecânica por penetração excessiva da agulha e resposta inflamatória aos componentes da vacina; no entanto, ainda não há um exame definitivo ou resultado quantificável.

Adverse reactions to vaccine injections are usually mild and incredibly rare in nature, but multiple cases of shoulder events including bursitis, generalized pain or decreased range of motion have been reported following routine vaccine administrations. These events are known as Shoulder Injury Related to Vaccine Administration or SIRVA. A systematic review of literature was performed to identify all published accounts of SIRVA. Twenty-seven papers reporting one or more accounts of SIRVA were identified. The most common vaccination involved was the Influenza vaccine. The most common symptoms were pain that began in 48 hours or less and loss of shoulder range of motion. The most common treatment modalities were physical therapy, corticosteroid
DOI https://doi.org/ $10.1055 / \mathrm{s}-0040-1719086$. ISSN 0102-3616. (c) 2020. Sociedade Brasileira de Ortopedia e Traumatologia. All rights reserved.

This is an open access article published by Thieme under the terms of the Creative Commons Attribution-NonDerivative-NonCommercial-License, permitting copying and reproduction so long as the original work is given appropriate credit. Contents may not be used for commercial purposes, or adapted, remixed, transformed or built upon. (https://creativecommons.org/ licenses/by-nc-nd/4.0/)

Thieme Revinter Publicações Ltda., Rua do Matoso 170, Rio de Janeiro, RJ, CEP 20270-135, Brazil 
injections and anti-inflammatory medication; but in some patients, surgery was

Keywords

- bursitis

- impingement, shoulder

- shoulder pain

- influenza vaccines required. Regardless of intervention, the vast majority of outcomes demonstrated improved pain and functional except in the occasions of nerve injury.

The etiology of SIRVA injuries has multiple possibilities including needle length, mechanical injury from needle overpenetration and the possibility of an immune inflammatory response from the vaccine components, but a unique definitive test or quantifiably result does not yet exist.

\section{Introdução}

A dor no ombro é um achado comum no ambiente de atenção primária; sua prevalência nos Estados Unidos é de 6,7\% a 26\%. ${ }^{1,2}$ Após o estabelecimento do Vaccine Adverse Event Reporting System (VAERS; Sistema de Notificação de Eventos Adversos de Vacinas dos Estados Unidos) em 1990, relatos de sintomas prolongados no ombro após vacinações foram documentados. $^{3,4}$ O Injection-Related Work Group (Grupo de Trabalho de Eventos Relacionados à Injeção) do Ministério da Saúde e Serviços Humanos e da Health Resources and Services Administration dos Centers for Disease Control dos Estados Unidos publicaram o Institute of Medicine Report de 2011, que gerou o documento Proposals for Updates to the Vaccine Injury Table (Propostas para Atualizações da Tabela de Lesões Vacinais). Este relatório sugere que a lesão em ombro relacionada à administração de vacinas (SIRVA, do inglês shoulder injury related to vaccine administration) se aplica quando o indivíduo imunizado, previamente sem dor ou disfunção do ombro, apresenta, até 48 horas após a vacinação, dor no ombro com limitação da amplitude de movimento. ${ }^{5}$

A SIRVA representa uma série complexa de lesões com início dos sintomas, tratamentos e desfechos relatados; foi adicionada à Vaccine Injury Compensation Table (Tabela de Compensação de Lesões Vacinais) publicada pela Health Resources and Services Administration. ${ }^{6}$ As estruturas supostamente acometidas são o manguito rotador, labrum, cápsula, bursa e músculo deltoide, com diagnósticos de bursite, rupturas do manguito rotador, capsulite adesiva, lesão condral, lesão nervosa e infecção. ${ }^{7-33} 0$ mecanismo mais comumente proposto é a penetração excessiva do músculo deltoide, que causa uma lesão mecânica e/ou imunemediada em resposta vacina e/ou adjuvantes; esses eventos são frequentemente relacionados a erros na técnica de injeção. ${ }^{7-33}$ Assim, o desfecho primário desta revisão foi a identificação de características únicas de SIRVA e os resultados clínicos. O desfecho secundário foi a avaliação do mecanismo proposto de lesão em relação às etiologias mais comumente sugeridas para a SIRVA (comprimento da agulha, técnica de vacinação e resposta autoimune) ${ }^{7-33} \mathrm{~A}$ hipótese é a identificação de achados diagnósticos únicos e a demonstração de desfechos clínicos passíveis de generalização, além de uma análise crítica dos fatores associados ao mecanismo proposto que dará orientações para evitar novas lesões em ombro.

\section{Métodos}

Uma revisão sistemática nos bancos de dados PubMed e Ovid MEDLINE foi realizada em 1 de fevereiro de 2020. Os termos de pesquisa "ombro" e "vacinação" foram utilizados em combinação. Os resultados da pesquisa foram obtidos de acordo com as orientações de Preferred Reporting Items for Systematic Reviews and Meta-Analyses ${ }^{34}$ (PRISMA) e uma lista de verificação PRISMA foi usada para análise desses resultados. Além disso, todas as citações presentes nos artigos foram verificadas. Estudos de nível I a V, publicados em inglês, foram considerados de acordo com os critérios de inclusão ${ }^{35}$ e quaisquer desfechos clínicos, incluindo, mas não se limitando a, diagnósticos de dor, redução da amplitude de movimento, infecção, lesão tendínea e lesão condral. Os critérios de exclusão foram estudos biomecânicos, artigos não relacionados a seres humanos, publicações em idioma que não o inglês, artigos de revisão sem novos casos relatados ou eventos tumorais após a vacinação. ${ }^{36}$

\section{Resultados}

Setenta e cinco estudos foram identificados (- Fig. 1). Após a seleção dos estudos sobre vacinação do ombro em humanos, restaram 44 artigos. Depois da exclusão daqueles não escritos em inglês, ficaram 39. A identificação de estudos cadavéricos, biomecânicos, incompletos ou sem dados clínicos excluiu outras 12 publicações. Os 27 artigos restantes foram analisados e revistos com cuidado.

No total, 56 relatos de dor, lesão ou infecção no ombro após a vacinação foram identificados. Os dados que mostram o tipo de vacinação, o tempo até o início dos sintomas, a duração do quadro clínico e a idade do paciente são mostrados na - Tabela 1. A faixa etária foi de 21 meses a 90 anos. A vacina mais comumente implicada foi influenza, representando $61 \%$ (34/56) dos casos. A segunda vacina mais relatada foi a vacina pneumocócica polivalente (PPV), com $14 \%(8 / 56)$ dos casos. O início exato dos sintomas não foi relatado em cinco casos, mas, em três deles, ocorreu em até 2 semanas após a vacinação. Nos 51 casos restantes, o início da dor ocorreu em 2 dias (48 horas) ou menos em $84 \%$ dos casos (43/51). A duração do quadro clínico não foi relatada em $38 \%$ (21/56) dos casos. Nos demais artigos, a duração do quadro clínico foi de 3 semanas ou menos em 63\% (22/35) dos casos. Os achados clínicos, tratamentos e desfechos relatados são demonstrados na - Tabela 2. Os métodos de tratamento 


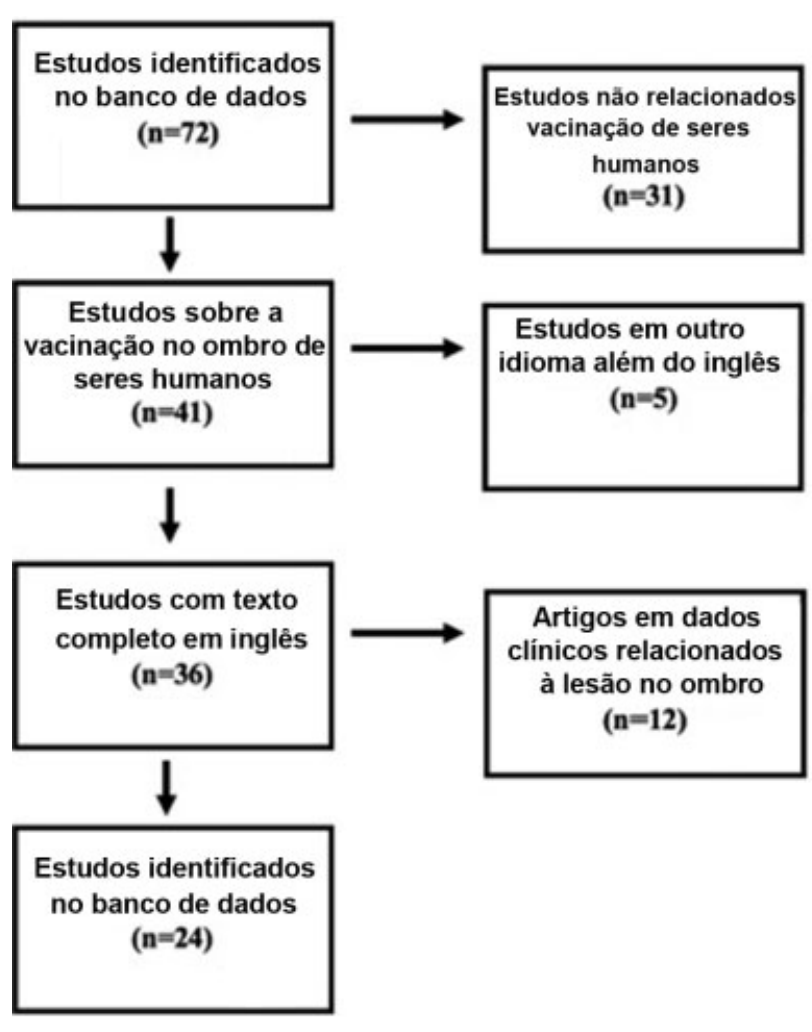

Fig. 1 Demonstração do processo de realização da revisão sistemática.

clínico foram relatados em todos os casos, exceto dois. As duas modalidades de tratamento mais comuns foram a fisioterapia, em 41\% (22/54) dos casos, e a injeção de corticosteroide (CSI), em 33\% (18/54). Doze (22\%) casos foram submetidos ao tratamento cirúrgico. Os desfechos clínicos de acompanhamento não foram relatados em 12 (21\%) casos. Dentre os relatos de desfechos clínicos, 30\% (13/44) dos casos apresentavam persistência dos sintomas além do período de acompanhamento. Nove dos 12 casos faziam parte de uma série em que a duração do quadro clínico até o tratamento não foi relatada. ${ }^{7}$ Os restantes $70 \%$ (31/44) dos relatos citaram melhora de funções e/ou sintomas.

\section{Discussão}

A coleta de dados sobre a disfunção de ombro relacionada à vacinação é recente, com apenas 56 relatos publicados. De acordo com a Tabela de Lesões Vacinais, ${ }^{6}$ os sintomas devem começar até 48 horas após a vacinação. Esta revisão demonstrou que $84 \%$ das publicações que relatam o tempo até o início da sintomatologia de fato atenderam aos critérios de 48 horas ou menos, sugerindo que parte da literatura não se enquadra na descrição de SIRVA da Tabela de Lesões Vacinais. Além disso, esse tempo até o início dos sintomas foi variável. Diversos casos foram relatados mais de três meses após a vacinação; o tempo mais longo relatado foi de 2 anos. $^{14,16,21,22,29}$ Vários estudos também citaram patologias como rupturas do manguito rotador e muitos casos ocorreram em pessoas com mais de 60 anos de idade. Diversos artigos mostraram que achados de ressonância magnética, como rupturas do manguito rotador, podem ser observados em indivíduos assintomáticos, com $50 \%$ de progressão a lacerações sintomáticas em um período médio de 2,8 anos. ${ }^{7,37-39}$ Assim, tempos maiores para desenvolvimento de sintomas, combinados à possível presença de doenças subjacentes, não permitem a demonstração de uma tendência; no entanto, a maioria dos estudos está em conformidade com a definição de menos de 48 horas.

Os achados mais comuns ao exame físico foram condizentes com o impacto e perda de amplitude de movimento do ombro. Apesar disso, não houve um único achado no exame físico que caracterizasse a SIRVA. Também não houve correlação clara entre um tipo de vacina e a gravidade do quadro clínico ou a duração dos sintomas. O tempo antes do início do tratamento foi variável. Esses tratamentos incluíram fisioterapia, injeções de corticosteroides, medicamentos anti-inflamatórios e/ou intervenções cirúrgicas. Os pacientes que começaram o tratamento médico específico nas primeiras 3 semanas de dor tenderam a apresentar resultados bons a excelentes, à exceção dos pacientes com lesão nervosa ou necessitaram de cirurgia. Os pacientes com lesão nervosa apresentaram sintomas persistentes e a progressão dos casos cirúrgicos foi mais longa. No entanto, a recuperação também foi excelente em muitos dos casos submetidos ao tratamento cirúrgico. De modo geral, isso demonstra que não houve um achado de exame físico específico em pacientes com SIRVA, mas, em indivíduos sem lesão neurológica, a recuperação quase completa ou total é o resultado mais comum. Além disso, os pacientes que começaram o tratamento dentro de 3 semanas do início dos sintomas geralmente tiveram bons resultados.

A análise de imagens de ressonância magnética (RM) demonstrou uma tendência. $8,10,12,13,21,24,26,32$ Salmon et al. ${ }^{8}$ descreve uma RM realizada dois dias após a vacinação que revela derrame glenoumeral, bursite subacromial, bursite subdeltoide e bursite subescapular. Uma RM magnética subsequente, 5 meses depois, revelou a regressão do derrame articular e a diminuição da bursite. Kuether et al. ${ }^{13}$ mostrou uma primeira RM com derrames menores na bursa subacromial e subdeltoide. As RMs subsequentes, obtidas aos 4 meses e 12 meses, demonstraram diminuição da bursite. Barnes et al. ${ }^{10}$ observaram derrame na bolsa subacromial em uma RM obtida 8 semanas após a vacinação. Uchida et al. ${ }^{26}$ também relataram bursite subacromial à RM após a vacinação. Atanasoff revisou 13 casos submetidos à RM e 69\% deles apresentaram coleções de fluidos na bursa ou tendinite do manguito rotador. Portanto, os primeiros achados à RM após um evento de SIRVA são correlacionados a alterações inflamatórias, como aumento de fluido, bursite e tendinite, mas os exames realizados meses depois podem não ser um método preciso de avaliação.

Como desfecho secundário desta revisão, o mecanismo associado à SIRVA foi avaliado. Sugere-se que esse mecanismo esteja relacionado à penetração excessiva do músculo deltoide, em que a agulha provoca uma lesão mecânica e/ou há desenvolvimento de uma resposta imune ao material injetado. Uma possível causa é a utilização de uma agulha longa. As diretrizes dos Centers for Disease Control and Prevention recomendam 
Tabela 1 Relatos de Lesões em Ombro Relacionadas à Vacinação

\begin{tabular}{|c|c|c|c|c|c|}
\hline AUTOR & $\begin{array}{l}\text { Tempo de } \\
\text { Aparecimento }\end{array}$ & $\begin{array}{l}\text { Duração do } \\
\text { Quadro Clínico }\end{array}$ & $\mathrm{N}$ & Vacinação & Idade \\
\hline Barnes et al. & $<24$ horas & 3 semanas & 1 & Influenza & 22 \\
\hline Shaikh et al. & $<1$ semana & 1 mês & 1 & Influenza & 46 \\
\hline Messerschmitt et al. & $<24$ horas & 3 semanas & 1 & Influenza & 46 \\
\hline Floyd et al. & $<24$ horas & 3 dias & 1 & PPV & 59 \\
\hline Kuether et al. & $<24$ horas & 4 semanas & 1 & Influenza & 48 \\
\hline Terreri et al. & Desconhecido & 1 semana & 1 & BCG & 21 meses \\
\hline Bodor et al. & 2 dias & $\begin{array}{l}5 \text { meses } \\
2 \text { meses }\end{array}$ & 2 & PPV e Influenza & 71 e 89 \\
\hline Cross et al. & $<24$ horas & 3 dias & 2 & PPV e dTpa & 82 e 23 \\
\hline Saleh et al. & $<24$ horas & $\begin{array}{l}6 \text { semanas } \\
3 \text { meses } \\
2 \text { anos }\end{array}$ & 3 & PPV e 2 Influenza & $67,30,69$ \\
\hline Hexter et al. & $<24$ horas & Imediata & 1 & Influenza & 50 \\
\hline Salmon et al. & $<24$ horas & 2 dias & 1 & Revaxis $^{\circledR}$ & 26 \\
\hline Okur et al. & $\begin{array}{l}<24 \text { horas: } 1 / 4 \\
\text { Desconhecido: } 3 / 4\end{array}$ & $\begin{array}{l}1-2 \text { semana: } 3 / 4 \\
2 \text { meses: } 1 / 4\end{array}$ & 4 & Todas Influenza & $66,59,39,36$ \\
\hline Cook et al. & $<24$ horas & 3 dias & 1 & Influenza & 76 \\
\hline Arias et al. & $\begin{array}{l}<24 \text { horas: } 3 / 8 \\
<1 \text { semana: } 3 / 8 \\
1-2 \text { meses: } 2 / 8\end{array}$ & Desconhecida & 8 & $\begin{array}{l}\text { 6/8 Influenza } \\
\text { 1/8 PPV } \\
\text { 1/8 Difteria, Toxoide tetânico }\end{array}$ & $22-89$ \\
\hline Anasoff et al. & $\begin{array}{l}<24 \text { horas: } 12 / 13 \\
4 \text { dias: } 1 / 13\end{array}$ & Desconhecida & 13 & $\begin{array}{l}8 \text { Influenza, } 2 \text { Td, } \\
2 \text { Tdap, } 1 \text { HPV }\end{array}$ & $22-83$ \\
\hline Degreef I e Debeer P & $\begin{array}{l}<24 \text { horas: } 2 / 3 \\
<1 \text { semana: } 1 / 3\end{array}$ & $\begin{array}{l}2 \text { meses } 2 / 3 \\
6 \text { meses } 1 / 3\end{array}$ & 3 & Hepatite A, Influenza, Tétano & $36,54,73$ \\
\hline McColgan BP e Borschke FA & $<24$ horas & $<24$ horas & 1 & PPV & 73 \\
\hline Bathia NA e Stitik T & $<24$ horas & 3 semanas & 1 & Influenza & 34 \\
\hline Shafer B e Burroughs $\mathrm{K}$ & $<24$ horas & 3 semanas & 1 & Influenza & 25 \\
\hline Uchida et al. & $<24$ horas & 3 semanas & 1 & HPV & 45 \\
\hline DeRogatis et al. & $<24$ horas & 1 semana & 1 & PPV & 90 \\
\hline Jotwani et al. & $<24$ horas & 2 semanas & 1 & Influenza & 61 \\
\hline Imran et al. & $<24$ horas & Desconhecida & 1 & Influenza & 73 \\
\hline Meirelles et al. & $<24$ horas & $1 \mathrm{dia}$ & 1 & Influenza, Difteria, Tétano & 67 \\
\hline Erickson et al. & Desconhecido & 2 semanas & 1 & Influenza & 51 \\
\hline Shahbaz et al. & $<24$ horas & 1 hour & 1 & Influenza & 34 \\
\hline Macomb et al. & $<24$ horas & $\begin{array}{l}<24 \text { horas } \\
4 \text { dias }\end{array}$ & 2 & PPV, Zoster & 69,84 \\
\hline
\end{tabular}

PPV, Vacina pneumocócica polivalente; BCG, bacilo de Calmétte-Guerin; dTpa, difteria, tétano e pertussis; Revaxis ${ }^{\circledR}$, difteria, tétano e poliomielite; HPV, papilomavírus humano; Tdap, tétano, difteria e pertussis.

uma agulha de 1 polegada $(2,5 \mathrm{~cm})$ em pacientes de todas as categorias, exceto duas. ${ }^{40} \mathrm{~A}$ primeira categoria se refere a mulheres com mais de 200 libras (cerca de $91 \mathrm{~kg}$ ) e homens com mais de 260 libras (118 kg), nos quais uma agulha de 1,5 polegadas $(3,8 \mathrm{~cm})$ é recomendada. A segunda categoria se refere a neonatos, nos quais se recomenda o uso de agulha de $5 / 8$ polegada $(1,6 \mathrm{~cm}) .{ }^{38}$ Poland et al. ${ }^{41}$ avaliaram a espessura da gordura deltoide por ultrassonografia e sugeriram agulhas de 1 polegada para homens, mas estratificaram a recomendação em mulheres como agulhas de $5 / 8$ polegadas naquelas com menos de $60 \mathrm{~kg}$, agulhas de 1 polegada naquelas com 60 a $90 \mathrm{~kg}$ e agulhas de 1,5 polegada naquelas com mais de $90 \mathrm{~kg}$. Um estudo semelhante foi realizado por Lippert et al., ${ }^{42}$ usando 250 séries de imagens, mas enfocando a penetração excessiva. Este estudo sugeriu que a penetração excessiva da agulha teria ocorrido em $11 \%$ dos pacientes com uma agulha de 5/8 polegadas, $55 \%$ dos pacientes com uma agulha de $7 / 8$ polegadas e $61 \%$ dos pacientes com uma agulha de 1 polegada. Esses autores sugeriram uma escala de peso que poderia eliminar as taxas de penetração excessiva, mas com um risco de $10 \%$ de 
Tabela 2 Achados Clínicos, Tratamentos e Desfechos

\begin{tabular}{|c|c|c|c|}
\hline AUTOR & Achados & Tratamento & Desfecho \\
\hline Barnes et al. & Dor no ombro, perda de ADM & FT & $\begin{array}{l}\text { Melhora da dor em } 11 \text { semanas } \\
\text { Resolução dos sintomas em } 16 \text { meses }\end{array}$ \\
\hline Shaikh et al. & $\begin{array}{l}\text { EMG - desnervação axonal do } \\
\text { deltoide e do supraespinhoso }\end{array}$ & Prednisolona oral & $\begin{array}{l}\text { Resolução da dor, mas persistência de fraqueza } \\
\text { "branda" em } 8 \text { meses }\end{array}$ \\
\hline $\begin{array}{l}\text { Messerschmitt } \\
\text { et al. }\end{array}$ & $\begin{array}{l}\text { Dor no ombro, perda de ADM, } \\
\text { lesão de cartilagem }\end{array}$ & Cirurgia - hemiartroplastia & Resolução da dor e dos sintomas em 3 anos \\
\hline Floyd et al. & Dor no ombro, perda de ADM & $\begin{array}{l}\text { Cirurgia - debridamento } \\
\text { artroscópico }\end{array}$ & Resolução da dor e dos sintomas em 12 semanas \\
\hline Kuether et al. & $\begin{array}{l}\text { Dor no ombro, osteonecrose } \\
\text { da cabeça do úmero }\end{array}$ & FT, AINEs orais & Resolução da dor e dos sintomas em 6 meses \\
\hline Terreri et al. & Dor no ombro, febre, osteíte & Antibióticos & Melhora dos sintomas 19 dias após antibióticos \\
\hline Bodor et al. & $\begin{array}{l}\text { Dor no ombro, perda de } \\
\text { ADM, tendinite }\end{array}$ & FT e CSI & Resolução da dor e dos sintomas em 5 e 6 meses \\
\hline Cross et al. & $\begin{array}{l}\text { Dor no ombro em ambos, } \\
\text { marcadores de infecção } \\
\text { em um paciente }\end{array}$ & $\begin{array}{l}\text { 1) Cirurgia - Debridamento } \\
\text { 2) FT e CSI }\end{array}$ & $\begin{array}{l}\text { Resolução da dor e dos sintomas em } 1 \text { mês } \\
\text { após a cirurgia e } 3 \text { meses após FT e CSI }\end{array}$ \\
\hline Saleh et al. & $\begin{array}{l}\text { Todos os } 3 \text { apresentavam } \\
\text { dor no ombro e perda de ADM }\end{array}$ & $\begin{array}{l}\mathrm{FT} 3 / 3 \\
\mathrm{CSI} 2 / 3\end{array}$ & $\begin{array}{l}\text { Resolução da dor e dos sintomas em } 50 \text { dias } \\
\text { em um paciente, desconhecida no segundo } \\
\text { e nula no terceiro paciente }\end{array}$ \\
\hline Hexter et al. & Dor no ombro & Debridamento cirúrgico & Resolução da dor e dos sintomas \\
\hline Salmon et al. & Dor e efusão no ombro & AINEs e CSI & Resolução da dor e dos sintomas em 5 meses \\
\hline Okur et al. & Dor no ombro & $\begin{array}{l}\text { AINEs } 3 / 4 \\
\text { Sem tratamento } 1 / 4\end{array}$ & $\begin{array}{l}\text { Resolução da dor e dos sintomas em } 5 \text { meses, } \\
33 \text { dias, } 5,5 \text { meses, } 2 \text { anos e } 2,5 \text { anos }\end{array}$ \\
\hline Cook et al. & Dor no ombro & $\mathrm{CSI}$ & Resolução da dor e dos sintomas em 1 meses \\
\hline Arias et al. & Dor no ombro & Desconhecido & Desconhecido \\
\hline Anasoff et al. & $\begin{array}{l}\text { Dor no ombro: } 13 / 13 \\
\text { Perda de ADM: } 11 / 13 \\
\text { Fraqueza: } 4 / 13\end{array}$ & $\begin{array}{l}\text { AINEs: } 8 / 13 \\
\text { CSI: } 8 / 13 \\
\text { FT: } 6 / 13 \\
\text { Cirurgia: } 4 / 13\end{array}$ & $\begin{array}{l}\text { Recuperação completa } 4 / 13 \\
\text { Sintomas residuais: } 9 / 13 \\
\text { Sintomas por pelo menos } 6 \text { meses } 13 / 13\end{array}$ \\
\hline $\begin{array}{l}\text { Degreef I e } \\
\text { Debeer P }\end{array}$ & $\begin{array}{l}\text { Todos os três apresentavam } \\
\text { dor no ombro e perda de ADM }\end{array}$ & $\begin{array}{l}\text { CSI: } 1 / 3 \\
\text { FT: } 3 / 3\end{array}$ & $\begin{array}{l}\text { Resolução da dor e dos sintomas em } 1 \text { mês, } \\
6 \text { semanas e } 3 \text { meses }\end{array}$ \\
\hline $\begin{array}{l}\text { McColgan BP e } \\
\text { Borschke FA }\end{array}$ & $\begin{array}{l}\text { Dor e aumento de } \\
\text { volume no ombro }\end{array}$ & & Melhora 2 semanas após a cirurgia \\
\hline $\begin{array}{l}\text { Bathia NA } \\
\text { e Stitik T }\end{array}$ & Dor no ombro & Desconhecido & Desconhecido \\
\hline $\begin{array}{l}\text { Shafer B e } \\
\text { Burroughs K }\end{array}$ & Dor no ombro e perda de ADM & Desconhecido & Desconhecido \\
\hline Uchida et al. & Dor no ombro & $\begin{array}{l}\text { Cirurgia - debridamento } \\
\text { artroscópico }\end{array}$ & $\begin{array}{l}\text { Resolução da dor e dos sintomas } \\
1 \text { ano após a cirurgia }\end{array}$ \\
\hline DeRogatis et al. & Dor e infecção no ombro & Debridamento cirúrgico & Melhora 2 semanas após a cirurgia \\
\hline Jotwani et al. & Dor no ombro & $\mathrm{CSI}$ & Melhora, mas sem relato do tempo transcorrido \\
\hline Imran et al. & Dor no ombro e fraqueza & FT & $\begin{array}{l}\text { Limitações da ADM às } 6 \text { semanas } \\
\text { de acompanhamento }\end{array}$ \\
\hline Meirelles et al & Dor no ombro e fraqueza & FT & $\begin{array}{l}\text { Recuperação significativa em } 1 \text { ano, retorno } \\
\text { da sensibilidade e função em } 31 \text { meses }\end{array}$ \\
\hline Erickson et al & Dor no ombro & $\begin{array}{l}\text { FT e CSI } \\
\text { Debridamento cirúrgico }\end{array}$ & Resolução da dor e dos sintomas em 1 ano \\
\hline Shahbaz et al. & Dor no ombro e perda de ADM & FT e AINEs & Melhora em 8 meses, mas persistência da dor \\
\hline Macomb et al. & Dor no ombro e perda de ADM & AINEs, FT, CSI & Resolução da dor e dos sintomas em 1 mês \\
\hline
\end{tabular}

Abreviaturas: ADM, Amplitude de movimento; FT, fisioterapia; EMG, eletromiografia; AINEs, Anti-inflamatórios não esteroidais; CSI, injeção de corticosteroide. 
penetração insuficiente. Cook et al. ${ }^{43}$ discutiram a importância de compreender o índice de massa corporal (IMC), demonstrando que, em todos os homens e mulheres com IMC inferior a 35, uma agulha de $25 \mathrm{~mm}$ de comprimento poderia ser utilizada com segurança; em mulheres com IMC superior a 35, no entanto, uma agulha de $32 \mathrm{~mm}$ seria necessária para penetração adequada. Atanasoff et al. ${ }^{7}$ examinaram 13 pacientes com dor persistente no ombro sem histórico de lesão local e indicaram a possível correlação entre SIRVA e o tamanho da agulha. De modo geral, esses estudos demonstraram que o uso de um único tamanho de agulha não é adequado, o que foi apoiado por análises de outros autores. ${ }^{44}$ Assim, é concebível que a penetração excessiva possa ocorrer em indivíduos com menor peso ou IMC, agulhas mais longas ou com combinação de maior comprimento da agulha e menor peso corporal/IMC. O comprimento apropriado da agulha, porém, deve diminuir significativamente o risco de penetração excessiva.

A técnica de vacinação também é comumente discutida. Muitos casos de SIRVA relatam a vacinação "muito alta" (menos de $3 \mathrm{~cm}$ da borda lateral do acrômio). $7,8,12,14,15,19,20,26$ Em um artigo, a bursa de dois indivíduos foi medida, demonstrando sua extensão de $3,5 \mathrm{~cm}$ a partir do acrômio em uma paciente do sexo feminino e $4 \mathrm{~cm}$ em um paciente do sexo masculino. ${ }^{14}$ Beals et al. ${ }^{45}$ examinaram a bursa de 17 ombros de cadáveres. Os autores observaram que a distância média do canto anterolateral do acrômio à cortina bursal posterior era de $2,8 \mathrm{~cm}$ e que as margens da bursa estavam sempre a $2 \mathrm{~cm}$ ou mais do canto anterolateral da superfície acromial da bursa. A realização mais distal da injeção pode impedir a ocorrência de lesões na bursa. Nos Estados Unidos, as recomendações técnicas de injeção sugerem sua realização 2 a 3 dedos (2 polegadas ou $5 \mathrm{~cm}$ ) abaixo do acrômio e declara que "para não causar lesão, a injeção não deve ser muito alta (perto do processo do acrômio) ou muito baixa"; 46 no entanto, o posicionamento excessivamente distal aumenta o risco de lesão no nervo axilar. Meirelles et al. ${ }^{30}$ relataram o caso de um homem de 67 anos que foi vacinado e imediatamente referiu dor e disfunção. Um estudo de condução nervosa revelou comprometimento do nervo axilar e a função só foi normalizada aos 31 meses. Imran et al. ${ }^{29}$ descreveram o caso de um homem de 73 anos com dor aguda após a vacinação. 0 exame físico e o exame muscular manual demonstraram deficiência funcional do deltoide e os autores sugeriram a lesão direta do nervo axilar como causa. Este paciente foi acompanhado por 6 semanas e apresentou melhora da função do ombro, mas déficits contínuos de amplitude de movimento. Assim, as vacinações no ombro com penetração excessiva são associadas ao risco de lesão da bursa em caso de localização superior e risco de lesão do nervo axilar em caso de localização inferior.

Por fim, a penetração da agulha além do músculo deltoide também gera o risco de injeção de vacina nos tecidos do ombro. A existência de uma resposta imune ao material injetado foi proposta por vários autores. ${ }^{7,8,12,13}$ Dumonde e Glynn ${ }^{47}$ demonstraram a capacidade de causar uma reação intra-articular em um modelo animal. ${ }^{48,49} \mathrm{Jasin}^{50}$ também utilizou um modelo em coelhos para análise do mecanismo de aprisionamento de imunecomplexos em tecidos colagenosos das articulações e descobriu que isso dependia da presença de anticorpos no espaço extravascular e da difusão de antígenos ou complexos solúveis neste espaço. Trollmo et al. ${ }^{51}$ avaliaram o sangue periférico de seis adultos saudáveis antes e 14 dias após a exposição ao antígeno. Esses autores demonstraram que o antígeno do vírus da influenza induz uma forte resposta sistêmica de anticorpos, mas não detectaram nenhuma diferença significativa no nível sistêmico observado após a injeção no espaço intra-articular em comparação à administração subcutânea.

A literatura traz diversos relatos de suspeita de reação inflamatória. Anasoff et al. ${ }^{7}$ sugeriu que uma injeção no espaço subacromial poderia causar uma reação. Salmon et al. ${ }^{8}$ descreveram um evento após a vacinação com demonstração de reação óssea à RM. Kuether et al. ${ }^{13}$ relataram uma mulher de 48 anos com sinais de osteonecrose na cabeça do úmero à RM imediatamente, 4 meses e 12 meses após a vacinação. Os autores afirmaram que uma relação causal direta não podia ser confirmada, mas propuseram uma resposta imune à injeção como possível etiologia da osteonecrose. Messerschmitt et al. ${ }^{12}$ discutiram o caso de um homem de 46 anos com dor no ombro imediatamente após a vacinação. 0 paciente foi levado para a cirurgia e as biópsias obtidas apresentavam células inflamatórias e tecido de granulação.

Embora a capacidade de causar uma resposta imune tenha sido indicada por dados obtidos em animais, ainda não há um estudo clínico definitivo que demonstre a associação quantitativa entre um antígeno e/ou adjuvante vacinal e uma inflamação imunemediada no ombro como causa de sintomatologia clínica prolongada. Isso é corroborado pelas declarações ao final de vários relatos de SIRVA. Kuether et al. ${ }^{13}$ afirmaram diversas vezes que o estabelecimento de um vínculo causal não era possível. Messerschmitt et al. ${ }^{12}$ sugeriram não terem certeza se as alterações condrolíticas eram anteriores ao evento. Uchida et at. ${ }^{26}$ foram além e afirmaram que as consequências da técnica de injeção inadequada não são conhecidas e que as amostras de biópsia obtidas 7 meses após a vacinação não puderam fornecer evidências conclusivas. Além disso, os diagnósticos, a duração e o tratamento dos casos relatados nesta revisão são heterogêneos, assim como os tipos de vacinas administradas. Dessa forma, os casos relatados não deram suporte quantitativo para uma resposta imune.

\section{Conclusão}

De modo geral, esta revisão demonstrou que, em pacientes sem lesão neurológica, a recuperação total ou quase completa é o resultado mais comum. Nenhuma característica exclusiva observada durante o exame físico foi identificada, mas a utilização precoce de RM pode ajudar o diagnóstico, demonstrando aumento do sinal de fluido e bursite. Por causa da heterogeneidade dos tratamentos instituídos, fisioterapia, CSI, AINEs ou cirurgia não podem ser recomendados de forma individual. Ao invés disso, o tratamento da patologia resultante deve ser recomendado conforme diretrizes baseadas em evidências para cada diagnóstico. Como os pacientes tratados em até 3 semanas após o início dos sintomas tendem a apresentar bons resultados, é possível recomendar que todos os pacientes 
com dor no ombro por mais de 14 dias após a vacinação procurem atenção médica imediata. Em relação ao comprimento da agulha, uma escala baseada em peso/IMC deve ser utilizada e as técnicas de vacinação devem evitar localizações superiores ao mesmo tempo em que minimizam o risco de lesão do nervo axilar. Por fim, ainda não se sabe se a lesão no ombro relacionada à administração da vacina, ou SIRVA, é um evento único. Aparentemente, para que SIRVA continue a ser termo descritivo, esses eventos teriam que ser exclusivos às vacinações e não simplesmente algo que pode acontecer em qualquer injeção com penetração excessiva da agulha. Portanto, mais dados são necessários para distinguir uma lesão mecânica de uma resposta imune.

\section{Conflito de Interesses}

O autor declara que não há conflito de interesses.

\section{Referências}

1 Cunningham LS, Kelsey JL. Epidemiology of musculoskeletal impairments and associated disability. Am J Public Health 1984;74(06):574-579

2 Luime JJ, Koes BW, Hendriksen IJM, et al. Prevalence and incidence of shoulder pain in the general population; a systematic review. Scand J Rheumatol 2004;33(02):73-81

3 Current Trends Vaccine Adverse Event Reporting System-United States. Available at: https://www.cdc.gov/mmwr/preview/mmwr html/00001804.htm. [Accessed March 1, 2019]

4 The National Childhood Vaccine Injury Act of 1986. Public Health Service Act ${ }^{* * *}$ 2125. (42 U.S.C. ${ }^{* * *}$ 300aa-25 (Supp. 1987))

5 Ryan T. 2011 Institute of Medicine (IOM) Report generated Proposals for Updates to the Vaccine Injury Table

6 Vaccine Injury Table. Available at: https://www.hrsa.gov/sites/ default/files/hrsa/vaccine-compensation/vaccine-injury-table.pdf. [Accessed March 1, 2019]

7 Atanasoff S, Ryan T, Lightfoot R, Johann-Liang R. Shoulder injury related to vaccine administration (SIRVA). Vaccine 2010;28(51): 8049-8052

8 Salmon JH, Geoffroy M, Eschard JP, Ohl X. Bone erosion and subacromial bursitis caused by diphtheria-tetanus-poliomyelitis vaccine. Vaccine 2015;33(46):6152-6155

9 Terreri MT, Yamada AF. Osteitis caused by BCG vaccination. Pediatr Radiol 2008;38(04):481

10 Barnes MG, Ledford C, Hogan K. A “needling" problem: shoulder injury related to vaccine administration. J Am Board Fam Med 2012;25(06):919-922

11 Shaikh MF, Baqai TJ, Tahir H. Acute brachial neuritis following influenza vaccination. BMJ Case Rep 2012;2012:1-2

12 Messerschmitt PJ, Abdul-Karim FW, Iannotti JP, Gobezie RG. Progressive osteolysis and surface chondrolysis of the proximal humerus following influenza vaccination. Orthopedics 2012;35 (02):e283-e286

13 Kuether G, Dietrich B, Smith T, Peter C, Gruessner S. Atraumatic osteonecrosis of the humeral head after influenza A-(H1N1) v2009 vaccination. Vaccine 2011;29(40):6830-6833

14 Bodor M, Montalvo E. Vaccination-related shoulder dysfunction. Vaccine 2007;25(04):585-587

15 Cook IF. Subdeltoid/subacromial bursitis associated with influenza vaccination. Hum Vaccin Immunother 2014;10(03):605-606

16 Saleh ZM, Faruqui S, Foad A. Onset of frozen shoulder following pneumococcal and influenza vaccinations. J Chiropr Med 2015;14 (04):285-289

17 Hexter AT, Gee E, Sandher D. Management of glenohumeral synovitis secondary to influenza vaccination. Shoulder Elbow 2015;7(02):100-103
18 Martín Arias LH, Sanz Fadrique R, Sáinz Gil M, Salgueiro-Vazquez ME. Risk of bursitis and other injuries and dysfunctions of the shoulder following vaccinations. Vaccine 2017;35(37):4870-4876

19 Floyd MW, Boyce BM, Castellan RM, McDonough EB. Pseudoseptic arthritis of the shoulder following pneumococcal vaccination. Orthopedics 2012;35(01):e101-e103

20 Cross GB, Moghaddas J, Buttery J, Ayoub S, Korman TM. Don't aim too high: Avoiding shoulder injury related to vaccine administration. Aust Fam Physician 2016;45(05):303-306

21 Okur G, Chaney KA, Lomasney LM. Magnetic resonance imaging of abnormal shoulder pain following influenza vaccination. Skeletal Radiol 2014;43(09):1325-1331

22 Degreef I, Debeer P. Post-vaccination frozen shoulder syndrome. Report of 3 cases. Acta Chir Belg 2012;112(06):447-449

23 McColgan BP, Borschke FA. Pseudoseptic arthritis after accidental intra-articular deposition of the pneumococcal polyvalent vaccine: a case report. Am J Emerg Med 2007;25(07):864.e1-864.e3

24 Bathia NA, Stitik T. "Influenza vaccine shoulder"-vaccination related traumatic injury to the infraspinatus: a case report [Poster presentation]. Assoc Acad Physiat Ann Meet 2010;. PM R 2009;1:S118

25 Shafer B, Burroughs K. Shoulder pain in a 25-year-old female following an influenza vaccination. Am Med Soc Sport Med 2010. Available at: https://www.amssm.org/shoulder_pain_in_a_25_year-csa

26 Uchida S, Sakai A, Nakamura T. Subacromial bursitis following human papilloma virus vaccine misinjection. Vaccine 2012;31 (01):27-30

27 DeRogatis MJ, Parameswaran L, Lee P, Mayer TG, Issack PS. Septic Shoulder Joint After Pneumococcal Vaccination Requiring Surgical Debridement. HSS J 2018;14(03):299-301

28 Jotwani V, Narducci DM. Pain in right shoulder · recent influenza vaccination - history of hypertension and myocardial infarction . Dx? J Fam Pract 2019;68(01):44-46

29 Imran M, Hayley D. Injection-induced axillary nerve injury after a drive- through Flu shot. Clin Geriatr 2013;21(12):

30 Meirelles H, Filho GRM. Axillary nerve injury caused by deltoid muscle intramuscular injection: case report. Rev Bras Ortop 2004;39(10):615-619

31 Erickson BJ, DiCarlo EF, Brause B, Callahan L, Hannafin J. Lytic Lesion in the Proximal Humerus After a Flu Shot: A Case Report. JBJS Case Connect 2019;9(03):e0248

32 Shahbaz M, Blanc PD, Domeracki SJ, Guntur S. Shoulder Injury Related to Vaccine Administration (SIRVA): An Occupational Case Report. Workplace Health Saf 2019;67(10):501-505

33 Macomb CV, Evans MO, Dockstader JE, Montgomery JR, Beakes DE. Treating SIRVA Early With Corticosteroid Injections: A Case Series. Mil Med 2020;185(1-2):e298-e300

34 Moher D, Liberati A, Tetzlaff J, Altman DG; PRISMA Group. Preferred reporting items for systematic reviews and metaanalyses: the PRISMA statement. PLoS Med 2009;6(07):e1000097

35 Obremskey WT, Pappas N, Attallah-Wasif E, Tornetta P 3rd, Bhandari M. Level of evidence in orthopaedic journals. J Bone Joint Surg Am 2005;87(12):2632-2638

36 Bilgili SG, Karadag AS, Ceylan MF, Calka O, Turktas U, Bulut G. The development of giant lipoma on the BCG vaccine caused scar. Cutan Ocul Toxicol 2012;31(01):70-73

37 Tempelhof S, Rupp S, Seil R. Age-related prevalence of rotator cuff tears in asymptomatic shoulders. J Shoulder Elbow Surg 1999;8 (04):296-299

38 Minagawa H, Yamamoto N, Abe H, et al. Prevalence of symptomatic and asymptomatic rotator cuff tears in the general population: From mass-screening in one village. J Orthop 2013;10(01):8-12

39 Yamaguchi K, Tetro AM, Blam O, Evanoff BA, Teefey SA, Middleton WD. Natural history of asymptomatic rotator cuff tears: a longitudinal analysis of asymptomatic tears detected sonographically. J Shoulder Elbow Surg 2001;10(03):199-203

40 Centers for Disease Control and Prevention. Dose, route, site, and needle size. Available at: www.immunize.org/catg.d/p3085.pdf. [Accessed March 22, 2019] 
41 Poland GA, Borrud A, Jacobson RM, et al. Determination of deltoid fat pad thickness. Implications for needle length in adult immunization. JAMA 1997;277(21):1709-1711

42 Lippert WC, Wall EJ. Optimal intramuscular needle-penetration depth. Pediatrics 2008;122(03):e556-e563

43 Cook IF, Williamson M, Pond D. Definition of needle length required for intramuscular deltoid injection in elderly adults: an ultrasonographic study. Vaccine 2006;24(07):937-940

44 Zuckerman JN. The importance of injecting vaccines into muscle. Different patients need different needle sizes. BMJ 2000;321 (7271):1237-1238

45 Beals TC, Harryman DT 2nd, Lazarus MD. Useful boundaries of the subacromial bursa. Arthroscopy 1998;14(05):465-470

46 How to Administer Intramuscular and Subcutaneous Vaccine Injections to Adults. Available at. http://www.immunize.org/catg.d/p2020A.pdf. [Accessed March 15, 2019]
47 Dumonde DC, Glynn LE. The production of arthritis in rabbits by an immunological reaction to fibrin. Br J Exp Pathol 1962;43(04): 373-383

48 Cooke TD, Jasin HE. The pathogenesis of chronic inflammation in experimental antigen-induced arthritis. I. The role of antigen on the local immune response. Arthritis Rheum 1972;15(04): 327-337

49 Cooke TD, Hurd ER, Ziff M, Jasin HE. The pathogenesis of chronic inflammation in experimental antigen-induced arthritis. II. Preferential localization of antigen-antibody complexes to collagenous tissues. J Exp Med 1972;135(02):323-338

50 Jasin HE. Mechanism of trapping of immune complexes in joint collagenous tissues. Clin Exp Immunol 1975;22(03):473-485

51 Trollmo C, Carlsten H, Tarkowski A. Intra-articular immunization induces strong systemic immune response in humans. Clin Exp Immunol 1990;82(02):384-389 